\section{REFERENCES}

1. Savoie M, Soloway MS, Kim SS, Manoharan M: A pelvic drain may be avoided after radical retropubic prostatectomy. J Urol. 2003; 170:112-4

2. Araki M, Manoharan M, Vyas S, Nieder AM, Soloway MS: A pelvic drain can often be avoided after radical
Retropubic prostatectomy- an update in 552 cases. Eur urol. 2006; 50: 1241-7

3. Sharma S, Kim HL, Mohler JL: Routine pelvic drainage not required after open or robotic radical prostatectomy. Urology 2007; 69: 330-3.

\title{
Re: Gynecologic-Tract Sparing Extra Peritoneal Retrograde Radical Cystectomy with Neobladder
}

\author{
Jagdeesh N. Kulkarni, S. Jamal Rizvi, U. Purushotthama Acharya, K. S. Shiva Kumar, \\ P. Tiwari
}

\section{Department of Urology, Bombay Hospital Institute of Medical Sciences, Mumbai, India}

Int Braz J Urol, 34: 180-190, 2008

To the Editor,

Bladder cancer is considered the most prevalent malignant tumor affecting male in Egypt. Orthotopic ileal neobladder is currently the preferred continent urinary diversion in suitable patients undergoing radical cystectomy for muscle-invasive bladder cancer and may be considered the gold standard with which other forms of diversion are compared. Incorporation of antireflux system in orthotopic ileal neobladder substitutes is important in protecting the upper urinary tract in all patients undergoing continent diversion with a reasonable life expectancy. If this were not important, why is it that normal human bladder anatomy has evolved with an effective antireflux mechanism? Indeed, many antireflux techniques have been developed but the multiplicity of these techniques suggests that an ideal solution has not been found. All antireflux anastmosis have an inherent risk of functional failure (1).

Patients with carcinoma in situ of the prostatic urethra, tumors near bladder neck or infiltrating the prostate, multifocal papillary tumors, history of upper tract tumors or positive margins on frozen section of the transected proximal urethra must be excluded. For these patients, continent cutaneous diversion using the same technique will be evolved soon. After radical cystectomy in females, both ureters are intussuscepted in modified Sigma pouch but most of the females now prefer orthotopic ileal neobladder (2).

The new technique, which prevents reflux, has several advantages compared with antireflux techniques: technical simplicity and the procedure is suitable for all types of ureters including normal, dilated, short and irradiated ureters. It allows a non obstructed unidirectional flow of urine with minimal rate of stenosis and/or surgical revision so; it can protect the upper urinary tract. The use of foreign material like staples or meshes is avoided and the antireflux system is constructed from a minimal length of bowel segment decreasing metabolic complications associated with malabsorption or resorption. The afferent short limb provides extra length to reach the ureter, a tension free anastomosis, no risks of ureteral angulation with neobladder filling, and the possibility to resect the ureter far above the bladder, thus avoiding ureteral ischemia and distal recur- 
rence. With intermediate follow up the S pouch ileal neobladder incorporating the new antireflux technique appears to be effective in preventing reflux of urine without significantly increasing the incidence of obstruction. The clinical and functional results of $S$ pouch ileal neobladder appear to be accepted. Despite these encouraging results prolonged follow up will be required to determine the durability and long term complications associated with orthotopic ileal neobladder.

\section{REFERENCES}

1. Hautmann RE: Urinary diversion: ileal conduit to neobladder. J Urol. 2003; 169: 834-42.

2. Denewer A: A low-pressure rectosigmoid pouch created by side-to-side anastomosis with a stapling technique and sigmoid colon intussusception as an antireflux procedure. Br J Urol. 1998; 81: 856-61.

Dr. Adel Denewer Department of Surgical Oncology Oncology Center, Mansoura University Mansoura, Egypt E-mail: adeldenewer@mans.edu.eg

\section{Men Reporting Lasting Longer with Hyperforin}

To the Editor,

Premature ejaculation (PE) is a common, embarrassing and significantly under treated medical condition that affects men and their partners. Dapoxetine, a new short lasting selective serotonin reuptake inhibitor (SSRI), has demonstrated clinical efficacy in clinical trials when used immediately before intercourse (1). However, Dapoxetine has not yet been approved by the regulatory agency in part because of concern of risk and benefit ration of a SSRI in this quality of life condition.

We became interested if Hypericum perforatum, a natural supplement that has demonstrated pharmacologically to inhibit serotonin reuptake and can be formulated to help men last longer during sexual intercourse. In a rat model of ejaculation duration, hyperforin extract from Hypericum perforatum can delay time to ejaculation $(2,3)$.

Ten male volunteers took the rapid release formulation of hyperforin (DeLithe Nutraceutical, In., Pittsburgh, PA) for 8 weeks and assessed their sexual intercourse duration and sexual satisfaction.

In 5 men with mean ejaculatory duration at baseline of less than 90 seconds (mean age 39 years old), 4 reported lasting longer - mean time to ejaculation before and after treatment, $58 \pm 12$ seconds to $131 \pm 23$ seconds, respectively $(\mathrm{p}<0.01)$. Equally interesting, the mean sexual intercourse duration increased from $266 \pm 39$ to $391 \pm 34 \mathrm{sec}-$ onds ( $\mathrm{p}=0.02$ ) after Hyperforin extract treatment in 3 of 5 men with ejaculation greater than 3 minutes at baseline (mean age 43 years old). No adverse effect on sexual function and no systemic side effects were reported.

Seven of 10 couples reported subjective global sexual satisfaction improvement for both the men and his partner after hyperforin. Five couples reported more frequent female orgasm. Rapid onset of action, ease of use and safety make hyperforin extract an option for men who wish to last longer (4). Prospective randomized studies are necessary to further evaluation the utility of hyperforin extract.

\section{REFERENCES}

1. Pryor JL, Althof SE, Steidle C, Rosen RC, Hellstrom WJ, Shabsigh R, at al.: Efficacy and tolerability of 